\title{
Self-energy effects in electronic Raman spectra of doped cuprates due to magnetic fluctuations
}

\author{
Roland Zeyher ${ }^{1}$ and Andrés Greco ${ }^{2}$ \\ ${ }^{1}$ Max-Planck-Institut für Festkörperforschung, Heisenbergstrasse 1, D-70569 Stuttgart, Germany \\ ${ }^{2}$ Departamento de Física, Facultad de Ciencias Exactas e Ingeniería and IFIR(UNR-CONICET), 2000 Rosario, Argentina
}

(Dated: August 3, 2018)

\begin{abstract}
We present results for magnetic excitations in doped copper oxides using the random phase approximation and itinerant electrons. In the $[1,0]$ direction the observed excitations resemble dispersive quasi-particles both in the normal and superconducting state similar as in recent resonant inelastic X-ray scattering (RIXS) experiments. In the $[1,1]$ direction the excitations form, except for the critical region near the antiferromagnetic wave vector $\mathbf{Q}=(\pi, \pi)$, only very broad continua. Using the obtained spin propagators we calculate electron self-energies and their effects on electronic Raman spectra. We show that the recently observed additional peak at about twice the pair breaking in $\mathrm{B}_{1 g}$ symmetry below $\mathrm{T}_{c}$ in $\mathrm{HgBa}_{2} \mathrm{CuO}_{4+\delta}$ can be explained as a self-energy effect where a broken Cooper pair and a magnetic excitation appear as final states. The absence of this peak in $\mathrm{B}_{2 g}$ symmetry, which probes mainly electrons near the nodal direction, is explained by their small selfenergies compared to those in the antinodal direction.
\end{abstract}

PACS numbers: 74.25.nd,74.25.Ha,74.72.Gh

Electronic Raman spectra in doped cuprates are dominated by a pair breaking peak in the superconducting state whereas in the normal state they are rather structureless. The main features of the spectra in the superconducting state can be explained within BCS theory using free quasi-particles. [1] On the other hand RIXS experiments have shown the existence of spin fluctuations in doped cuprates in form of dispersive, broad but well-defined excitations, both in the normal and the superconducting state. 2, 3] The interaction between spin fluctuations and electrons gives rise to self-energies which have been used to explain, for instance, the observed kinks in the electronic dispersion in many high- $\mathrm{T}_{c}$ superconductors. [4 6] Self-energy effects should also be present in electronic Raman spectra causing for instance the recently observed broad peak above the pair breaking peak in $\mathrm{HgBa}_{2} \mathrm{CuO}_{4+\delta}$. [7] It is the purpose of this Letter to calculate the properties of spin fluctuations, the resulting self-energies and their effects on Raman scattering and to compare the results with recent experiments. [2, 7] Our calculations are based on an itinerant picture of magnetism and will be limited to the optimally doped and overdoped region to avoid unsolved problems with the existence and nature of the pseudogap in the underdoped regime.

The Raman susceptibility can be written in the superconducting state as, [8]

$$
\begin{array}{r}
\chi_{\alpha}\left(i \nu_{m}\right)=\frac{1}{N_{c}} \sum_{\mathbf{k}} \gamma_{\alpha}^{2}(\mathbf{k})\left(\Pi_{11,11}\left(\mathbf{k}, i \nu_{m}\right)+\Pi_{22,22}\left(\mathbf{k}, i \nu_{m}\right)\right. \\
\left.-\Pi_{12,21}\left(\mathbf{k}, i \nu_{m}\right)-\Pi_{21,12}\left(\mathbf{k}, i \nu_{m}\right)\right),(1)
\end{array}
$$

The indices $i, j, \ldots$ assume the values 1 or $2, \nu_{m}$ denotes a bosonic Matsubara frequency, $f$ the Fermi function, and $N_{c}$ is the number of primitive cells. Assuming a square lattice and using the lattice constant as the length unit $\gamma_{\alpha}(\mathbf{k})$ stands for the form factors $\gamma_{1}(\mathbf{k})=t\left(\cos k_{x}+\cos k_{y}\right)+4 t^{\prime} \cos k_{x} \cos k_{y}, \quad \gamma_{3}(\mathbf{k})=$ $t\left(\cos \left(k_{x}-\cos k_{y}\right), \gamma_{4}(\mathbf{k})=-4 t^{\prime} \sin k_{x} \sin k_{y}\right.$, corresponding to the representations $\mathrm{A}_{1 g}, \mathrm{~B}_{1 g}$, and $\mathrm{B}_{2 g}$ of the point group $\mathrm{D}_{4 h}$, respectively. $t$ and $t^{\prime}$ are effective nearest and second-nearest neighbor hopping amplitudes.

Using the Nambu representation the inverse of the $2 \times 2$ electron Green's function matrix $G\left(k, i \omega_{n}\right)$ is

$$
\begin{array}{r}
G^{-1}\left(\mathbf{k}, i \omega_{n}\right)=\left(i \omega_{n}-\Sigma_{+}\left(\mathbf{k}, i \omega_{n}\right)\right) \tau_{0} \\
-\left(\epsilon(\mathbf{k})+\Sigma_{-}\left(\mathbf{k}, i \omega_{n}\right)\right) \tau_{3}-\Delta(\mathbf{k}) \tau_{1} .
\end{array}
$$

$\tau_{1}$ and $\tau_{3}$ are Pauli matrices, $\tau_{0}$ the $2 \times 2$ unit matrix, and $\omega_{n}$ a fermionic Matsubara frequency. $\Sigma_{ \pm}\left(\mathbf{k}, i \omega_{n}\right)$ stands for $\left(\Sigma_{11}\left(\mathbf{k}, i \omega_{n}\right) \mp \Sigma_{11}\left(-\mathbf{k},-i \omega_{n}\right)\right) / 2$, where $\Sigma_{11}\left(\mathbf{k}, i \omega_{n}\right)$ is the $(1,1)$ component of the $2 \times 2$ self-energy matrix. $\epsilon(\mathbf{k})$ is the electron energy

$$
\epsilon(\mathbf{k})=-2 t\left(\cos k_{x}+\cos k_{y}\right)-4 t^{\prime} \cos k_{x} \cos k_{y}-\mu,
$$

and the spectral function $A_{i j}$ is defined by $-1 / \pi \operatorname{Im} G_{i j}(\mathbf{k}, \omega+i \eta)$. The self-energy $\Sigma$ is calculated from the Fock diagram with a bosonic propagator describing spin fluctuations. To simplify the calculation we do not consider the non-diagonal part of $\Sigma$ assuming that it has already been taken into account by the phenomenological gap parameter $\Delta(\mathbf{k})$. Assuming also that the frequency-independent Fock term has been accounted for in the phenomenological hoppings $t$ and $t^{\prime}$ the frequency-dependent part of $\Sigma_{11}$ is given by [4]

$$
\begin{array}{r}
\Sigma_{11}\left(\mathbf{k}, i \omega_{n}\right)= \\
-\frac{T}{2 N_{c}} \sum_{\mathbf{q}, m} J^{2}(\mathbf{q}) \chi\left(\mathbf{q}, i \nu_{m}\right) G_{11}^{(0)}\left(\mathbf{k}+\mathbf{q}, i \omega_{n}+i \nu_{m}\right) .
\end{array}
$$


$\chi\left(\mathbf{k}, i \nu_{m}\right)$ is the zz component of the spin susceptibility which assumes in the RPA the form [4, 9]

$$
\chi\left(\mathbf{k}, i \nu_{m}\right)=\frac{\chi^{(0)}\left(\mathbf{k}, i \nu_{m}\right)}{1-J(\mathbf{k}) \chi^{(0)}\left(\mathbf{k}, i \nu_{m}\right)} .
$$

$\chi^{(0)}\left(\mathbf{q}, i \nu_{m}\right)$ represents the bare spin bubble. $J(\mathbf{k})$ is equal to $2 J\left(\cos k_{x}+\cos k_{y}\right)$ where $J$ is the Heisenberg constant. Since self-energy effects in Raman spectra are expected to be rather small we may use $G_{11}^{(0)}$ instead of $G_{11}$ in Eq.(5).

In the following calculations we used the parameters $t^{\prime} / t=-0.25$ and $\mu / t=-0.9$ corresponding to the doping $\delta=0.15$, where $t$ is the effective nearest-neighbor hopping amplitude and about $0.15 \mathrm{eV}$ in optimally doped YBCO. 10] We expect a similar value in the case of $\mathrm{HgBa}_{2} \mathrm{CuO}_{4+\delta}$. $J$ was determined so that the experimental value of $0.056 \mathrm{eV}$ [1] for the magnetic resonance energy in the superconducting state was reproduced yielding $J / t=1.30$. The bare order parameter $\Delta$ was fixed to $\Delta / t=0.45$ which reproduces roughly the position of the observed pair breaking peak in $\mathrm{B}_{1 g}$ symmetry in the sample with the highest $\mathrm{T}_{c}$ of $94 \mathrm{~K}$ of Ref. [7].

An important ingredient for the calculation of $\Sigma$ is the dynamical spin susceptibility $\chi(\mathbf{k}, \omega)$. Its imaginary part $\chi^{\prime \prime}(\mathbf{k}, \omega)$ has been discussed extensively in the literature [9] in conjunction with the resonant mode, i.e., in the superconducting state and near the antiferromagnetic wave vector $\mathbf{Q}$. We extend these calculations to wave vectors throughout the Brillouin zone (BZ) and also to the normal state. Such calculations are interesting in view of recent RIXS measurements [2] which cover a more extended region. The upper and lower diagrams in Fig. 1 show $\chi^{\prime \prime}(\mathbf{k}, \omega)$ along the $[1,1]$ direction in the normal and the superconducting state, respectively. For wave vectors away from $\mathbf{Q}$ (and also from the origin $(0,0)$ ) the spectra are extremely broad and structureless, exhibiting often several flat maxima. Only the center of gravity of the lines indicates an increase with momentum up to around the middle of the zone and then a pronounced decrease towards $\mathbf{Q}$. In the normal state the curve for momentum $\mathbf{Q}$ shows a well-pronounced critical peak due to the proximity of the antiferromagnetic instability. In the superconducting state this peak moves to somewhat higher energies and becomes the resonant peak with a finite width due to our employed damping constant $\eta=0.1$. Being a bound state inside the gap it attracts a huge amount of spectral weight. This holds to a lesser degree even for the momentum $3 \mathbf{Q} / 4$ where the structureless continuum in the normal state transforms into a well-pronounced peak in the presence of superconductivity.

$\chi^{\prime \prime}(\mathbf{k}, \omega)$ looks quite different in the $[1,0]$ direction as illustrated in Fig. 2. It shows both in the normal and the superconducting state well-defined and narrow peaks which disperse as a function of momentum similar as the spin waves of a Heisenberg model. However, in our case
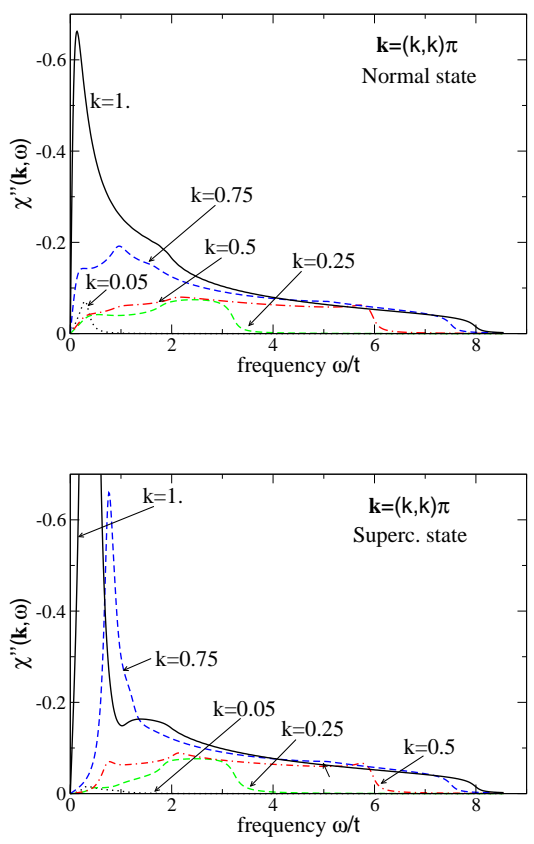

FIG. 1. (Color online) $\chi^{\prime \prime}(\mathbf{k}, \omega)$ along the $[1,1]$ direction in the normal (upper diagram) and superconducting (lower diagram) state at $\mathrm{T}=0$.

the sharp peaks do not describe collective excitations. This means that the sharp peaks in Fig. 2 do not correspond to poles of the denominator of $\chi(\mathbf{k}, \omega)$ but are due to dynamical nesting properties of $\chi^{(0)}(\mathbf{k}, \omega)$. The energy scale of the magnetic dispersion is in our case determined by the electron band dispersion, i.e., by the kinetic energy of the electrons and not by the Heisenberg constant $J$. This is especially clear at the point $(\pi, 0)$ : In the RPA $J$ drops out in $\chi$ and the energy of the magnetic exitation is soley due to the kinetic energy of the electrons. Using different band parametrizations (for instance, those given in Table I of Ref. [10]) we found very similar results as in Figs. 1-2 showing that these results are generic for doped cuprates.

$\chi^{\prime \prime}(\mathbf{k}, \omega)$ has recently been determined by RIXS measurements along the $[1,0]$ direction for several copper oxides and successfully interpreted within a local-moment model including damping. 2] Fig. 2 and its comparison with Ref. 2] shows that the nature of the magnetic excitations, their dispersion and their band width are similar in the itinerant and in the local moment approach, though the underlying phyics is quite different. One reason for this is that $J$ and the effective hopping $t$ have very similar values of about $150 \mathrm{meV}$. In the $[1,1]$ direction the two approaches may show larger differences because the itinerant model predicts in this case rather broad and irregular line shapes except for a small critical region around $(\pi, \pi)$.

Fig. 3 depicts the imaginary part of $\Sigma(\mathbf{k}, \omega)$ (upper diagram) and the spectral function $A_{11}(\mathbf{k}, \omega)$ (lower diagram) for the momentum on the Fermi line along the 

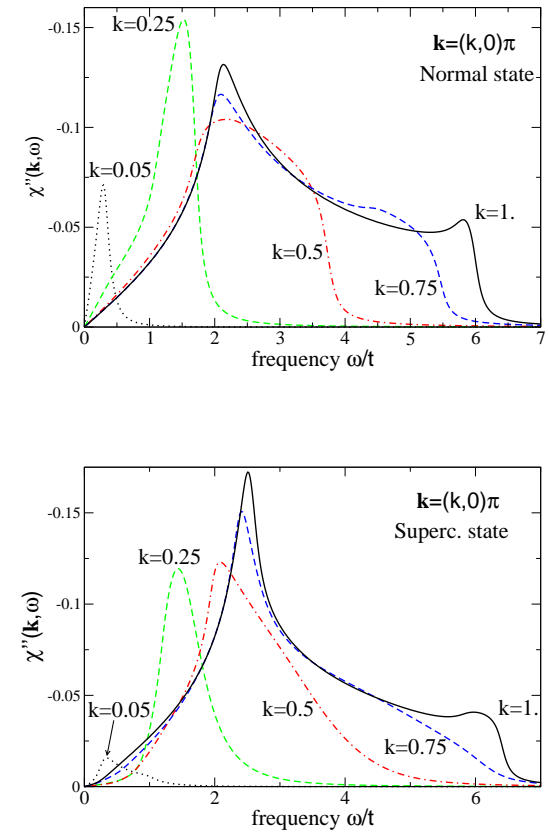

FIG. 2. (Color online) $\chi^{\prime \prime}(\mathbf{k}, \omega)$ along the $[1,0]$ direction in the normal (upper diagram) and superconducting (lower diagram) state at $\mathrm{T}=0$.

$[1,1]$ direction. In the normal state $\operatorname{Im} \Sigma(\mathbf{k}, \omega)$ is zero at $\omega=0$, increases away from zero due to scattering of electrons with spin fluctuations, and finally decreases due to the finite band width. In the superconducting state electron scattering is impeded by the gap leading to a depletion of spectral weight around $\omega=0$ in $\operatorname{Im} \Sigma$. On the other hand superconductivity causes a large shift of spectral weight of spin fluctuations towards low energies, especially near $(\pi, \pi)$. Momentum transfers of about $(\pi, \pi)$ lead in general to scattered electrons far away from the Fermi line for the considered intial electron momentum. The result is the superconductivity-induced peak around $\omega / t \sim 2$., i.e., well away from the Fermi energy. The spectral function $A_{11}(\mathbf{k}, \omega)$ consists of a simple peak at $\omega=0$ without any sidebands.

Fig. 4 shows the same as Fig. 3 but for the electron momentum on the Fermi line along the $(\pi, 0)-(\pi, \pi)$ direction. Now there are important electronic transitions between the surroundings of $(\pi, 0)$ and $(0, \pi)$ with momentum transfers near $(\pi, \pi)$, i.e., where spin fluctuations have a large spectral weight and low energies. In the normal state the peak due to critial scattering near $(\pi, \pi)$ in the upper part of Fig. 1 causes the low-lying peaks in the imaginary part of $\Sigma(\mathbf{k}, \omega)$, as seen in Fig. 4. In the superconducting state the same scattering processes are even much stronger due to the appearance of the resonant mode which carries a large amount of spectral weight. As a result the distance between the two low-lying peaks in $\operatorname{Im} \Sigma(\mathbf{k}, \omega)$ in the normal state increases due to the su-
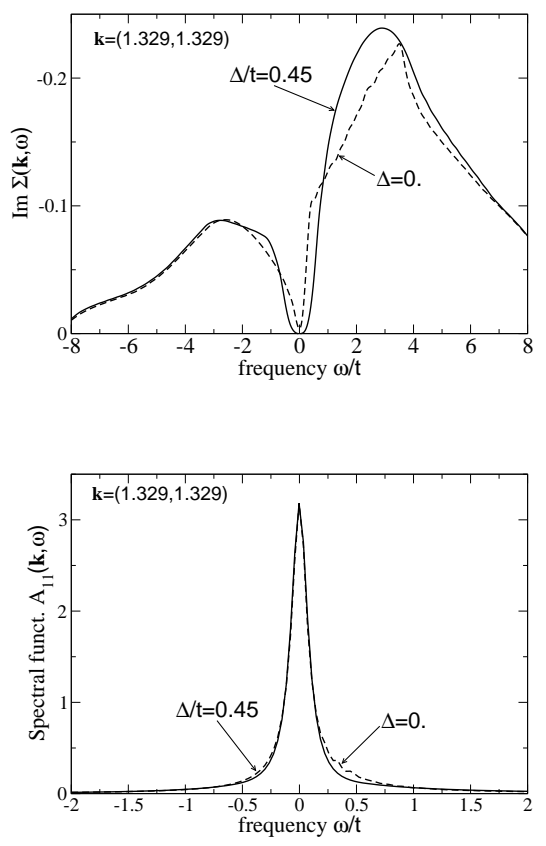

FIG. 3. Imaginary part of the self-energy (upper diagram) and spectral function (lower diagram) for the momentum on the Fermi line in $[1,1]$ direction in the normal and superconducting state.

perconducting gap and at the same time the peaks gain a lot of strength. The corresponding spectral functions reflect the low-lying peaks in form of sidebands, which are strongly pronounced especially in the superconducting state. The position of the sideband peaks would be in the absence of dispersion the sum of half of the superconducting gap plus the resonance frequency. The dispersion of electrons and of the resonance peak causes an additional shift to the positions shown in the figure.

Finally, Fig. 5 gives our results for the Raman intensity $-\operatorname{Im} \chi_{\alpha}(\omega+i \eta)$ where $\alpha$ denotes one of the three symmetries $\mathrm{B}_{1 g}, \mathrm{~B}_{2 g}$ and $\mathrm{A}_{1 g}$. In each case the dashed line corresponds to the normal and the solid line to the superconducting state. Let us first discuss the $\mathrm{B}_{1 g}$ spectrum (upper part in Fig. 5). In the free case, defined by $\Sigma=0$, the Raman intensity would consist in the normal state of a $\delta$-function at $\omega=0$ (not shown in the figure) and a Lorentzian-like pair breaking peak at the unrenormalized energy $2 \Delta$. Turning on $\Sigma(\mathbf{k}, \omega)$ the $\delta$ function is replaced by a continuum in the normal state which increases rather abruptly from zero at zero frequency to a finite value and then decreases very slowly towards higher frequencies. This is the result of electron scattering from spin fluctuations. The solid curve, referring to the superconducting state and $\mathrm{B}_{1 g}$ symmetry, exhibits two maxima. The first one is due to the renormalized pair breaking peak, the second one due to the additional emission of a magnetic excitation, for instance, the resonance mode. The position of the additional high-frequency peak can also be estimated from 

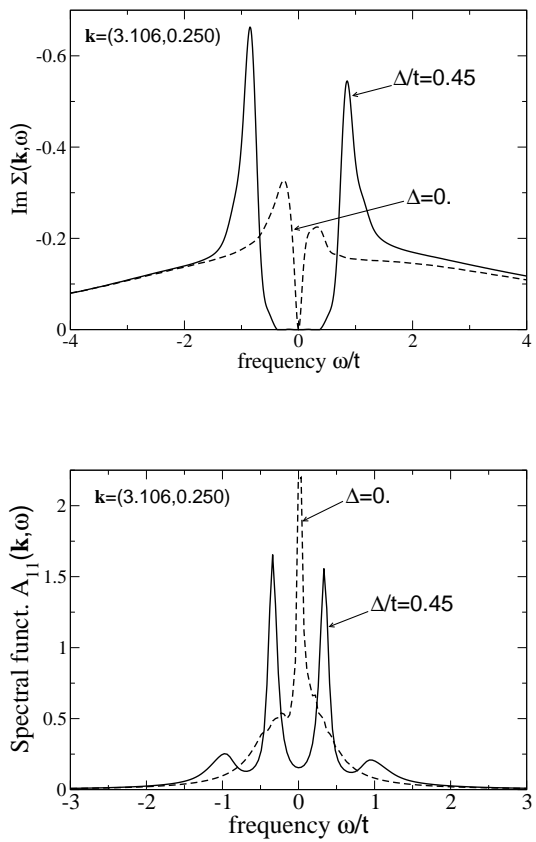

FIG. 4. Imaginary part of the self-energy (upper diagram) and spectral function (lower diagram) for the momentum on the Fermi line in $(\pi, 0)-(\pi, \pi)$ direction in the normal and superconducting state.

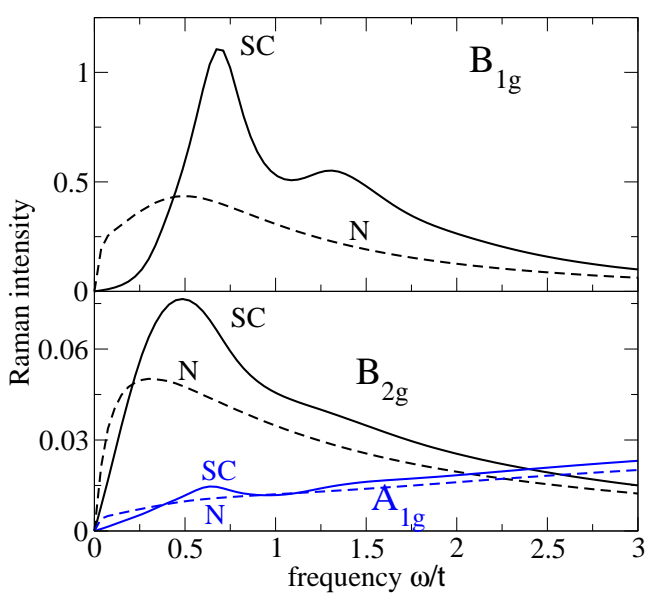

FIG. 5. (Color online) Raman spectra with $\mathrm{B}_{1 g}$ (upper part), $\mathrm{B}_{2 g}$ and $\mathrm{A}_{1 g}$ symmetries (lower part) in the normal (dashed lines) and superconducting (solid lines) state.

the spectral function $A_{11}$. According to Eq.(1) the Raman intensity at frequency $\omega$ is essentially be obtained as a folding of two spectal functions displaced by the frequency $\omega$. As a result the pair breaking peak arises from tansitions between the two dominating peaks in Fig. 4. A smaller peak is expected from transitions between one of the large peaks and a sideband peak yielding a frequency of about 1.3 in good agreement with Fig. 5. The ratio of the positions of the two peaks is about 1.9 which is near the experimental value. [7] We stress that the position and the intensity of the second peak in this spectrum is not fitted but a consequence of the parameters $J$ and $\Delta$ determined such that the observed resonance frequency and the pair breaking peak are at least approximately reproduced. Using the electronic parameters of Ref. [4] the additional peak in the $\mathrm{B}_{1 g}$ spectrum is practically absent. The reason for this is that the self-energy in this case is substantially smaller than in our case. The difference in the magnitude of the self-energies can at least partially be traced back to the large difference in the resonance frequencies, namely $56 \mathrm{meV}$ in $\mathrm{HgBa}_{2} \mathrm{CuO}_{4+\delta}$ and $40 \mathrm{meV}$ in YBCO for nearly optimally doped samples.

The $\mathrm{B}_{2 g}$ spectrum (contained in the lower diagram in Fig. 5) is in the normal state similar to that of the $\mathrm{B}_{1 g}$ symmetry except that its intensity is smaller by one order of magnitude. In the superconducting state it shows a pair breaking peak but no additional peak at higher frequencies, in agreement with experiment. [12] The reason for this can easily be understood: The form factor in the $\mathrm{B}_{2 g}$ symmetry weights electrons near the nodal direction strongly. There the gap and also the self-energy are small. As a result the pair breaking moves somewhat to lower frequencies compared to the $\mathrm{B}_{1 g}$ symmetry and no additional peak appears as a result of self-energy effects. For completeness we also show the $\mathrm{A}_{1 g}$ spectrum (lower diagram in Fig. 5) where Coulomb screening has been taken into account. Here we encounter the wellknown and hitherto unsolved problem that its intensity is too small by about one order of magnitude compared to experiment. 13] Thus we will exclude it from our discussion.

We used in our treatment a broken Cooper pair times a spin fluctuation as final states. Using the same interactions one may also consider a competing process where two spin fluctuations or paramagnons appear as final states. [7] For zero or very small doping such a localized description is certainly appropriate whereas in the optimal or overdoped regime a description in terms of itinerant electrons should be a better choice. For instance, it allows easily to understand why the additional high-energy peak shows a strong dependence on $T_{c}$ similar like the resonance mode 7] or why its existence is tied to the existence of the pair breaking peak and the depletion region below it. It also has been argued 14] that two-magnon processes are neglegible small in the $\mathrm{B}_{1 g}$ and $\mathrm{B}_{2 g}$ configurations and thus may be discarded in these cases.

In summary, we have shown that an itinerant picture of magnetism may account for the observed dispersive magnetic excitations in $[1,0]$ direction in doped cuprates and also for the observed additional structure above the pair breaking peak in electronic Raman scattering in $\mathrm{HgBa}_{2} \mathrm{CuO}_{4+\delta}$.

The authors thank M. Le Tacon for a critical reading of the manuscript. They also acknowledge useful dis- 
cussions with Y. Li, M. Le Tacon, A. Schnyder and B. Keimer. R.Z. and A.G. are grateful to the Dep. de Física (Rosario) and the MPI-FKF (Stuttgart), respectively, for hospitality and financial support.

[1] For a recent review see T. Devereaux and R. Hackl, Rev. Mod. Phys. 79, 175 (2007).

[2] M. Le Tacon et al., Nature Physics 7, 725 (2011).

[3] M.P.M. Dean et al., arXiv:1212.3714.

[4] R. Zeyher and A. Greco, Phys. Rev. B 64, 140510(R) (2001).
[5] T. Dahm et al., Nature Physics 5, 217 (2009).

[6] D. Manske, Theory of Unconventional Superconductors, Springer Tracts in Modern Physics 202, (Springer, Berlin (2004)).

[7] Yuan Li et al., Phys. Rev. Lett. 108, 227003 (2012).

[8] R. Zeyher and A. Greco, Phys. Rev. Lett. 89, 177004 (2002).

[9] M. Eschrig and M.R. Norman, Phys. Rev. B67, 144503 (2003).

[10] M.R. Norman, Phys. Rev. B 63, 092509 (2001).

[11] G. Yu et al., Phys. Rev. B 81, 064518 (2010).

[12] Yuan Li, private communication.

[13] Y. Gallais et al., Phys. Rev. Lett. 88, 17740 (2002), and references therein.

[14] F. Venturini, U. Michelucci, T.P. Devereaux, and A.P. Kampf, Phys. Rev. B62, 15204 (2000). 